\title{
O que a Neurociência tem a nos dizer (ou ainda não) sobre o processamento da metáfora?
}

\author{
What does Neuroscience have to tell us (or not yet) about metaphor processing?
}

\author{
Kári Lúcia Forneck \\ Universidade do Vale do Taquari, Lajeado, RS, Brasil
}

\begin{abstract}
Resumo: As pesquisas com neuroimagem acerca do processamento da metáfora apresentam achados distintos, o que implica dificuldades na formulação de um paradigma teórico de base neurológica da metáfora. Neste estudo, argumentamos que a falta de clareza de aspectos conceituais sobre a metáfora sob uma perspectiva linguística implica diversidades no desenho dos experimentos e incongruências nos achados. Para sustentar esse argumento, apresentamos uma análise qualitativa de um corpus composto por 20 experimentos com neuroimagem acerca do processamento da metáfora sob dois aspectos: os fundamentos conceituais de metáfora e os achados obtidos nos experimentos. Verificamos que a ausência de um diálogo entre Neurociência e Linguística sobre as propriedades da metáfora resulta numa delimitação problemática de um padrão de processamento da metáfora sob um viés neurológico.
\end{abstract}

Palavras-chave: Processamento da metáfora; Neurociência; fMRI; Meta-análise

\begin{abstract}
Neuroimaging research on the processing of metaphor presents different findings, which implies difficulties in formulating a theoretical paradigm of the metaphor in a neurological perspective. In this study, we argue that the lack of clarity of conceptual aspects about metaphor from a linguistic perspective implies diversities in the experiments design and incongruities in the findings. To support this argument, we present a qualitative meta-analysis of a corpus composed of $20 \mathrm{fMRI}$ experiments on the processing of metaphor, under two aspects: the conceptual foundations of metaphor and the findings obtained in the experiments. As a result, we find that the absence of a dialogue between Neuroscience and Linguistics about the metaphor properties results in a problematic delimitation of a metaphor processing pattern under a neurological bias.
\end{abstract}

Keywords: Processing metaphor; Neuroscience; fMRI; Meta-analysis

\section{Considerações iniciais}

As pesquisas com neuroimagem, em especial a ressonância magnética funcional (fMRI), apesar de relativamente recentes, têm acrescentado novas possibilidades de verificação de hipóteses linguísticas. Em contrapartida, como apontam Laird et al. (2009) e Laird et al. (2011), há muitos dados à disposição, com achados particularmente distintos, o que dificulta a prospecção de padrões cerebrais. A existência de bancos de dados, como BrainMap, que têm o propósito de compor metaanálises dos achados das pesquisas das funções cerebrais humanas, evidencia a importância de se desenharem padrões neuroanatômicos para as funções do cérebro.
Em relação às pesquisas acerca do processamento da metáfora, por exemplo, há achados distintos, o que implica dificuldades na formulação de um paradigma teórico de base neurológica para o tema. Além disso, características cognitivas individuais - como a manipulação de conhecimentos prévios -, bem como aspectos contextuais e intenções do falante são, segundo Ferstl (2012), variáveis complexas no desenho de experimentos a serem testados com neuroimagem.

Por essa complexa configuração, os achados de estudos com neuroimagem a respeito do processamento da metáfora apontam para diferenças anatômicas no recrutamento da circuitaria cortical tanto no hemisfério esquerdo (HE), quanto no hemisfério direito (HD). 
Há razões que podem explicar essas distinções, tanto em função dos procedimentos experimentais adotados e dos correlatos neuronais analisados, quanto em função dos fundamentos epistemológicos subjacentes. No presente estudo, pretendemos argumentar como a falta de clareza de aspectos conceituais sobre a metáfora sob uma perspectiva linguística implica diversificação no desenho dos experimentos com neuroimagem e, consequentemente, incongruências nos achados. Para tanto, o texto divide-se em duas seções: na primeira, apresentam-se hipóteses acerca do processamento da metáfora comumente referenciadas nos estudos com neuroimagem; na seção seguinte, trataremos da apresentação e da análise qualitativa de um corpus composto por 20 experimentos sobre o processamento da metáfora sob dois aspectos: as relações conceituais que subjazem os experimentos e os achados a que chegaram os pesquisadores.

\section{Modelos neurológicos do tratamento da metáfora}

Três hipóteses fundamentam boa parte das pesquisas com fMRI sobre o processamento cognitivo da metáfora: graded salience hypothesis (GIORA, 1997; GIORA et al., 2000), coarse-coding hypothesis (BEEMAN, 1998; JUNG-BEEMAN, 2005) e dynamic spillover hypothesis (JUST; VARMA, 2007; PRAT; MASON; JUST, 2011).

$\mathrm{Na}$ perspectiva da Graded salience hypothesis (GSH), um significado é entendido como saliente quando pode ser recuperado diretamente do léxico armazenado na memória. Em contrapartida, um significado é não-saliente quando precisa ser acessado por processos inferenciais de integração (cf. GIORA et al., 2000).

Essa hipótese se fundamenta na premissa de que uma metáfora, se frequentemente usada, passa a ser reconhecida como convencional e lexicalizada. Nesse caso, seu significado não literal será considerado a mais saliente significação em qualquer contexto.

Segundo a GSH, significados mais salientes, familiares, conhecidos ou prototípicos são processados em primeiro plano, independentemente do contexto. O papel do contexto seria o de bloquear os sentidos incompatíveis. Propriedades como convencionalidade, frequência, familiaridade e prototipicalidade são variáveis da composição dos significados salientes dos itens lexicais.

Do ponto de vista da anatomia do cérebro, significados salientes são processados antes de significados não-salientes, especialmente no hemisfério esquerdo (HE), que, portanto, tem um papel crucial no processamento de metáforas mais convencionais, cujo significado já se cristalizou. Giora e colegas (2000) sugerem que o envolvimento do hemisfério direito (HD) varia em função do grau de saliência das propriedades que compõem os significados das metáforas, como familiaridade e convencionalidade, por exemplo. O HD será acessado quando a interpretação de significados nãosalientes requerer a configuração de relações semânticas não-salientes ou mais distantes (cf. GIORA et al., 2000).

No âmbito da Coarse-coding hypothesis ( $\mathrm{CCH}$ ), assume-se que o processamento de metáforas implica assimetria cerebral. Para Jung-Beeman (2005), o processamento semântico depende de três etapas: a ativação, a integração e a seleção.

A etapa da ativação pode ocorrer bilateralmente, principalmente no giros temporal posterior médio e superior (área de Wernicke). O HE, com ativação focada e forte, é mais sensível aos sentidos dominantes, literais e contextualmente relevantes, enquanto o HD, com ativação mais difusa e fraca, é mais sensível a relações semânticas distantes (cf. BEEMAN, 1998). A integração semântica corresponde ao processo de busca de coerência entre diferentes ativações semânticas e depende do giro bilateral temporal superior anterior, sulco temporal superior, giro temporal médio e polo temporal. O córtex temporal do HD é mais ativado quando a integração semântica ocorre em razão de enunciados não literais e mais complexos. A seleção é a etapa em que são escolhidos entre os diferentes significados acessados aqueles mais relevantes para o contexto. Durante esse processo, ativa-se principalmente $o$ giro frontal inferior esquerdo.

Em relação ao processamento da metáfora, os estudos fundamentados nessa hipótese defendem o argumento de que o HD tem papel essencial especialmente na compreensão de metáforas criativas, aquelas em que os elementos linguísticos em relação de significado têm poucas propriedades semânticas em comum. Mais especificamente, ao HD caberiam as funções de acessar as categorias semânticas dos elementos em comparação e selecionar as mais adequadas ao contexto, enquanto ao HE caberia a função de correlacionar os conceitos evocados e integrá-los conceitualmente (cf. FERSTL, 2012, p. 59).

Por fim, segundo a Dynamic spillover hypothesis $(D S H)$, regiões homólogas às da linguagem no HD são recrutadas em uma variedade de situações linguísticas, quando o esforço cognitivo é maior que os recursos disponíveis pelo HE. Segundo Prat, Mason e Just (2011), resíduos de ativação neuronal "transbordam" para regiões homólogas no $\mathrm{HD}$, quando a compreensão não for alcançada pelo HE.

Essa hipótese prediz que o envolvimento do HD irá variar de acordo com a dificuldade de leitura e, principalmente, em razão das particularidades cognitivas dos indivíduos (cf. PRAT; MASON; JUST, 2011). Just e Varma (2007, p. 154) argumentam que o pensamento é o produto da atividade de múltiplas áreas do cérebro que 
colaboram mutuamente em uma ampla rede cortical. Cada área cortical, com capacidade limitada de recursos, pode assumir múltiplas funções cognitivas e, inversamente, múltiplas funções cognitivas podem recrutar diferentes áreas. A topologia da rede cortical se altera dinamicamente durante os processos cognitivos, adaptando-se à limitação de recursos de diferentes áreas corticais e às exigências funcionais de cada tarefa.

Em relação ao processamento da metáfora, estudos de fMRI que se fundamentarem nessa hipótese encontrarão achados particularmente associados às características cognitivas individuais dos participantes, já que a compreensão de metáforas poderá variar dependendo da capacidade e da habilidade linguística de cada indivíduo e do esforço demandado pela tarefa de compreensão.

Apresentadas as hipóteses que norteiam estudos com neuroimagem, apresenta-se a seguir uma meta-análise qualitativa de estudos sobre o processamento da metáfora, a partir da qual se tecem algumas considerações a respeito das relações conceituais que subjazem os experimentos e dos achados a que chegaram os pesquisadores.

\section{Composição do corpus de pesquisa e análise dos achados}

Para compor o corpus dessa pesquisa, foram os artigos científicos com estudos experimentais com neuroimagem que tratavam de verificar como a metáfora é processada. Para dar conta dessa tarefa, inserimos os termos de busca metáfora e fMRI em sites de busca de artigos, como PubMed, ScienceDirect, Scielo, Capes e Google Acadêmico.

Nesse levantamento foram encontrados 56 artigos, tabulados, num primeiro momento, a partir do ano de sua publicação. No refinamento da seleção, foram mantidos aqueles nos quais: a) os participantes da pesquisa eram adultos (não idosos) saudáveis; b) os experimentos visavam a descrever os correlatos neurais do processamento de metáforas nominais primordialmente, e não de ironias, expressões idiomáticas e sarcasmo; c) as metodologias previam a compreensão de metáforas e não a produção de enunciados com metáforas. Decorre desse refinamento a seleção de 20 artigos, que são apresentados a seguir:

Tabela 1. Estudos da metáfora nominal com fMRI

\begin{tabular}{|c|c|c|c|}
\hline & Autores & Título & Ano \\
\hline 1 & $\begin{array}{l}\text { RAPP, Alexander M.; LEUBE, Dirk T.; ERB, Michael; GRODD, Wolfgang; } \\
\text { KIRCHER, Tilo T. J. }\end{array}$ & Neural correlates of metaphor processing & 2004 \\
\hline 2 & MASHAL, Nira; FAUST, Miriam; HENDLER, Talma & $\begin{array}{l}\text { The role of the right hemisphere in processing nonsalient metaphorical } \\
\text { meanings: application of principal components analysis to fMRI data }\end{array}$ & 2005 \\
\hline 3 & LEE, Susan S.; DAPRETTO, Mirella & $\begin{array}{l}\text { Metaphorical vs. literal word meanings: } F M R I \text { evidence against a selective } \\
\text { role of the right hemisphere }\end{array}$ & 2006 \\
\hline 4 & $\begin{array}{l}\text { STRINGARIS, Argyris K.; MEDFORD, Nicholas C.; GIORA, Rachel; } \\
\text { GIAMPIETRO, Vincent C.; BRAMMER, Michael J.; DAVID, Anthony S. }\end{array}$ & $\begin{array}{l}\text { How metaphors influence semantic relatedness judgments: the role of the } \\
\text { right frontal cortex }\end{array}$ & 2006 \\
\hline 5 & MASHAL, Nira; FAUST, Miriam; HENDLER, Talma; JUNG-BEEMAN, Mark & $\begin{array}{l}\text { An } \mathrm{fMRI} \text { investigation of the neural correlates underlying the processing of } \\
\text { novel metaphoric expressions }\end{array}$ & 2007 \\
\hline 6 & $\begin{array}{l}\text { AHRENS, Kathleen; LIU, Ho-Ling; LEE, Chia-Ying; CONG, Shu-Ping; } \\
\text { FANG, Shin-Yi; HSU, Yuan-Yu }\end{array}$ & $\begin{array}{l}\text { Functional MRI of conventional and anomalous metaphors in Mandarin } \\
\text { Chinese }\end{array}$ & 2007 \\
\hline 7 & SHIBATA, Midori; ABE, JJun-ichi; TERAO, Atsushi; MIYAMOTO, Tamaki & $\begin{array}{l}\text { Neural mechanisms involved in the comprehension of metaphoric and literal } \\
\text { sentences: an fMRI study }\end{array}$ & 2007 \\
\hline 8 & $\begin{array}{l}\text { RAPP, Alexander M.; LEUBE, Dirk T.; ERB, Michael; GRODD, Wolfgang; } \\
\text { KIRCHER, Tilo T. J. }\end{array}$ & $\begin{array}{l}\text { Laterality in metaphor processing: lack of evidence from functional magnetic } \\
\text { resonance imaging for the right hemisphere theory }\end{array}$ & 2007 \\
\hline 9 & $\begin{array}{l}\text { STRINGARIS, Argyris K.; MEDFORD, Nicholas C.; GIAMPIETRO, Vincent; } \\
\text { BRAMMER, Michael J.; DAVID, Anthony S. }\end{array}$ & $\begin{array}{l}\text { Deriving meaning: distinct neural mechanisms for metaphoric, literal, and } \\
\text { non-meaningful sentences }\end{array}$ & 2007 \\
\hline 10 & $\begin{array}{l}\text { YANG, Fanpei Gloria; EDENS, Jennifer; SIMPSON, Claire; KRAWCZYK, } \\
\text { Daniel. }\end{array}$ & Differences in task demands influence the hemispheric lateralization & 2009 \\
\hline 11 & MASHAL, Nira; FAUST, Miriam; HENDLER, Talma; BEEMAN, Mark & An fMRI study of processing novel metaphoric sentences & 2009 \\
\hline 12 & SCHMIDT, Gwenda; SEGER, Carol & $\begin{array}{l}\text { Neural correlates of metaphor processing: the roles of figurativeness, } \\
\text { familiarity and difficulty }\end{array}$ & 2009 \\
\hline 13 & $\begin{array}{l}\text { BAMBINI, Valentina; GENTILI, Claudio; RICCIARDI, Emiliano; } \\
\text { BERTINETTOA, Pier Marco; PIETRINI, Pietro }\end{array}$ & $\begin{array}{l}\text { Decomposing metaphor processing at the cognitive and neural level } \\
\text { through functional magnetic resonance imaging }\end{array}$ & 2011 \\
\hline 14 & DIAZ, Michele T.; BARRET, Kyle T.; HOGSTROM, Larson J. & The influence of sentence novelty and figurativeness on brain activity & 2011 \\
\hline 15 & DIAZ, Michele T.; HOGSTROM, Larson J. & $\begin{array}{l}\text { The influence of context on hemispheric recruitment during metaphor } \\
\text { processing }\end{array}$ & 2011 \\
\hline 16 & PRAT, Chantel; MASON, Robert; JUST, Marcel A. & $\begin{array}{l}\text { An fMRI investigation of analogical mapping in metaphor comprehension: the } \\
\text { influence of context and individual cognitive capacities on processing demands }\end{array}$ & 2012 \\
\hline 17 & $\begin{array}{l}\text { SHIBATA, Midori; TOYOMURA, Akira; MOTOYAMA, Hiroki; ITOH, Hiroaki; } \\
\text { KAWABATA, Yasuhiro; ABE, Jun-ichi }\end{array}$ & $\begin{array}{l}\text { Does simile comprehension differ from metaphor comprehension: a } \\
\text { functional MRI study }\end{array}$ & 2012 \\
\hline 18 & $\begin{array}{l}\text { CARDILLO, Eileen R.; WATSON, Christine E.; SCHMIDT, Gwenda L.; } \\
\text { KRANJEC, Alexander; CHATTERJEE, Anjan }\end{array}$ & From novel to familiar: tuning the brain for metaphors & 2012 \\
\hline 19 & SUBRAMANIAM, Karuna; BEEMAN, Mark; FAUST, Miriam; MASHAL, Nira & $\begin{array}{l}\text { The repetition paradigm enhancement of novel metaphors and suppression } \\
\text { of conventional metaphors in the left inferior parietal lobe }\end{array}$ & 2012 \\
\hline 20 & SUBRAMANIAM, Karuna; BEEMAN, Mark; FAUST, Miriam; MASHAL, Nira & $\begin{array}{l}\text { Positively valenced stimuli facilitate creative novel metaphoric processes by } \\
\text { enhancing medial prefrontal cortical activation }\end{array}$ & 2013 \\
\hline
\end{tabular}

Fonte: A autora. 
O corpus foi analisado sob dois aspectos: a natureza dos fundamentos linguísticos sobre a metáfora, assumidos como ponto de partida para os experimentos, e os resultados e as conclusões a que chegaram seus autores. A seguir, apresentamos as análises dos achados.

\subsection{Do ponto de vista dos fundamentos sobre metáfora}

Apesar de ser um objeto de estudo prioritariamente linguístico-cognitivo ${ }^{1}$, boa parte dos artigos selecionados não apresentou fundamentos de base linguística para definição da metáfora. Em boa parte deles os pesquisadores se propuseram, apenas, a apresentar uma referência a estudos prévios que constroem a metáfora sob o ângulo das Neurociências e, em alguns casos, sob os fundamentos da Psicolinguística, numa interface cognitiva da metáfora.

Dos experimentos selecionados, nove estudos tomaram como ponto de partida a graded salience hypothesis (GSH), assumindo, portanto, que metáforas devem ser entendidas como elementos linguísticocognitivos com propriedades semânticas, entre as quais, convencionalidade, frequência, familiaridade e prototipicalidade, que são decisivas na composição dos significados. Nesse caso, como já dissemos, o significado mais saliente sempre será processado em primeiro plano, independentemente do contexto. Portanto, nesses estudos, o envolvimento do HD se justifica em função do grau de saliência dos significados que compõem a metáfora.

Essa estruturação teórica se deu em Mashal, Faust e Hendler (2005), Lee e Dapretto (2006), Stringaris et al. (2006), Ahrens et al. (2007), Mashal et al. (2007 e 2009), Diaz, Barret e Hogstrom (2011), Diaz e Hogstrom (2011), Subramaniam et al. (2012). Desses estudos, quatro mencionaram também a coarse-coding hypothesis (CCH). Entre eles estão Stringaris et al. (2006), Mashal et al. (2009), Diaz, Barret e Hogstrom (2011) e Subramaniam et al. (2012).

Com relação à coarse-coding hypothesis $(\mathrm{CCH})$, houve seis experimentos que assumiram a hipótese de que o recrutamento do HD se dá em função da seleção de relações semânticas mais distantes, em ativação mais difusa e fraca. É o caso dos estudos de Stringaris et al. (2006), Mashal et al. (2009), Schmidt e Seger (2009), Diaz, Barret e Hogstrom (2011) e Subramaniam et al. (2012; 2013). Como anteriormente mencionado, quatro estudos referenciaram também a GSH: Stringaris et al. (2006), Mashal et al. (2009), Diaz, Barret e Hogstrom (2011) e Subramanian et al. (2012).

Apenas o estudo de Prat, Mason e Just (2012) assumiu à dynamic spillover hypothesis (DSH) e a

\footnotetext{
A metáfora, por sua natureza complexa, pode ser objeto de estudo em outras áreas, como a Filosofia e a Psicologia, por exemplo.
}

pressuposição de que características individuais são decisivas na compreensão de enunciados com metáforas.

Cardillo e colegas (2012), por sua vez, assumiram a Carreira da Metáfora $^{2}$ (cf. GENTNER; BOWDLE, 2008) como pano de fundo teórico para a delimitação do experimento. $\mathrm{O}$ design do experimento tomou como ponto de partida a hipótese de que a compreensão da metáfora demanda dois processos: a comparação e a categorização. Esses mecanismos cognitivos foram traduzidos para correlatos neurais nesse estudo.

Bambini e colegas (2011) estabeleceram uma relação entre Neurociência e Pragmática, assumindo que há demandas cognitivas adicionais que precisam ser levadas em consideração para o estudo do processamento da metáfora. Para os autores, a compreensão da metáfora envolve subfunções distribuídas na circuitaria cerebral. Entre elas, há o fato de que deve haver fatores pragmáticos envolvidos nesse processo, entre os quais, um componente conceitual, que prevê a integração de informações linguísticas com o conhecimento de mundo do indivíduo que se encontra diante de um enunciado metafórico; um componente de atenção, que garante o monitoramento das memórias a serem acessadas para a construção do conceito metafórico; e um componente de reconhecimento das intenções do outro. Todas essas variáveis implicam, segundo os autores, aumento do recrutamento do sistema cognitivo e, por extensão, do sistema neurológico. Não haveria, portanto, como estudar a metáfora sem levar em consideração aspectos da Pragmática. O experimento proposto por Bambini et al. (2011) parece-nos original, por ser o único que tratou da metáfora numa perspectiva interdisciplinar entre Neurociência e Linguística.

Seis experimentos não assumiram como ponto de partida hipóteses de base linguística ou psicolinguística sobre a metáfora: Rapp et al. (2004 e 2007), Shibata et al. (2007) e Shibata et al. (2012), Stringaris et al. (2007) e Yang et al. (2009). Stringaris et al. (2007) e Shibata et al. (2012) fizeram alusão aos estudos sobre o processamento de metáforas como categorias de significação (cf. GLUCKSBERG; KEYSAR, 1990; GLUCKSBERG et al., 1997; GLUCKSBERG, 2008), mas não desenharam os experimentos sob esse paradigma. O mesmo ocorreu com relação à Carreira da Metáfora (cf. GENTNER; BOWDLE, 2008), que é mencionada por Shibata et al. (2012), sem, no entanto, servir como fundamento para a estruturação do experimento. Em outras palavras, os

\footnotetext{
A hipótese da Carreira da Metáfora pressupõe que é possível que sejam criadas categorias metafóricas, que são constituídas à medida que uma determinada metáfora é empregada repetidamente ao longo do tempo. De acordo com essa hipótese, as metáforas sofrem um gradual processo de abstração e de convencionalização, desde que tenham sido empregadas pela primeira vez como metáforas criativas (cf. GENTNER; BOWDLE, 2008, p. 116)
} 
teóricos apresentados serviam apenas como referência prévia para ilustrar como a metáfora é tratada nesse campo de pesquisa, mas não tiveram ressonância no desenho do experimento, nem nas análises dos achados.

Todos os seis experimentos partiram de hipóteses $a d$ hoc, construídas pelo levantamento de estudos prévios com outras técnicas, como, por exemplo, divided visual field technique, pósitron emission tomography (PET) e event related potential (ERP), além de estudos com participantes com algum tipo de lesão no cérebro. Esses estudos fornecem evidências de padrões de processamento cognitivo a partir de outras metodologias de coleta de dados.

Para ilustrar a complexidade dos conceitos envolvidos, citamos Stringaris e colegas (2007). Para os autores, a habilidade de categorização, tal como pensada por Glucksberg e Keysar (1990), e a propriedade da composicionalidade da metáfora, como sugerida por Fodor e Pylyshyn (1988), são os argumentos teóricos que justificam os achados. Entretanto, o instrumento não foi desenhado metodologicamente seguindo essas premissas. Trata-se, portanto, de um paradigma abdutivo, diferente do que costuma acontecer em pesquisas com fMRI.

Em outras palavras, se metáforas fossem assumidas como categorias de significação, como ao final do texto os autores argumentaram, o desenho do experimento deveria também ter sido estruturado sob essa premissa. Assim, para uma sentença como (1), empregada no experimento, seria necessário levar em consideração de algum modo a categoria coragem, para verificar que correlatos neurais são ativados quando categorias de significação como essa são conceitualmente construídas pelos sujeitos participantes da testagem.

\section{(1) Alguns homens são leões.}

É o caso, também, de Rapp et al. (2007) que propuseram aos participantes que avaliassem metáforas como (2-4) sob dois aspectos, a metaforicidade e a conotação:

(2) As palavras de amor são sons de harpa.

(3) A reunião com o diretor foi um calvário.

(4) O despertador é uma tortura.

Nessa testagem, os participantes tinham de decidir se sentenças como (2-4) comportavam sentido literal ou metafórico e, num segundo momento, se tinham conotação positiva ou negativa. Sob que critérios pode-se atribuir conotação positiva ou negativa para uma sentença como (4), por exemplo, é uma pergunta que não pôde ser respondida no estudo. Parece-nos que, se a metáfora tem apenas duas propriedades, ser ou não metáfora e ter ou não conotação positiva, o conceito de metáfora assumido remonta aos primeiros estudos linguísticos sobre linguagem metafórica, entendida como um adorno linguístico, sem levar em consideração todo um conjunto de estudos sobre a metáfora que justamente problematizam essa definição. Em nosso entender, a restrição do conceito de metáfora limita também os achados do experimento.

O problema da valência - positiva ou negativa - das metáforas também aparece em Mashal (2009):

For example, the literal sentence "Flowers wilted in the garden's flowerbed" was rated as a sentence with negative meaning (2.26 on the valence scale), whereas the novel metaphoric sentence "The sunrise wears the trees' veil" was rated as a sentence with positive meaning (3.5 on the valence scale) (MASHAL et al., 2009 , p. 8$)^{3}$.

Caso assumamos que há uma gradação na significação de metáforas, como propuseram os autores ao solicitar aos participantes que avaliassem em escala o significado das metáforas, entendemos não ser possível assumir, então, que tenham significado positivo (são metáforas) ou negativo (não são metáforas).

Aparentemente, familiaridade, convencionalidade, frequência, figuratividade, plausibilidade, entre outras, foram propriedades da metáfora exploradas de diferentes maneiras nos estudos selecionados. Como sugere Kasparian (2013), é necessária uma certa prudência na análise dos dados, porque "one major point is that there is a lack of clear consensus in the literature with respect to what exactly constitutes a metaphor" (KASPARIAN, 2013, p. 15) ${ }^{4}$.

Esses dados ilustram que, do ponto de vista teórico, ainda não se pode construir uma abordagem da metáfora de base neurocientífica com potencial explanatório abrangente o suficiente para desenhar a metáfora como um objeto de estudo com propriedades linguísticas, cognitivas e neurológicas, de modo a descrever a circuitaria envolvida no processamento e explicar como e por que os padrões encontrados têm essas caracte-

rísticas.

Além do mais, se a cada novo experimento uma hipótese ad hoc é testada sem um compromisso teórico prévio, ou seja, sem que o objeto de investigação seja desenhado teoricamente no escopo de uma abordagem

\footnotetext{
Por exemplo, a frase literal "Flores murcharam no canteiro de flores do jardim" foi classificada como uma sentença com significado negativo (2,26 na escala de valência), enquanto a nova sentença metafórica "O nascer do sol usa o véu das árvores" como sentença com significado positivo (3,5 na escala de valência) (MASHAL et al., 2009:8, tradução nossa).

4 [...] um ponto importante é que há uma falta de consenso claro na literatura em relação ao que exatamente constitui uma metáfora (KASPARIAN, 2013:15, tradução nossa).
} 
teórica, é provável que os achados reflitam ad hoc a proposta escolhida.

\subsection{Do ponto de vista dos achados}

Apresentamos na subseção anterior divergências em termos de fundamentos teóricos sobre a metáfora. Evidentemente, essas diferenças todas implicam também diferenças nos resultados. Como Kasparian (2013) argumenta, o papel do HD no processamento da metáfora ainda permanece inconclusivo, apesar da quantidade de achados que se tem à disposição. Isso porque há estudos favoráveis à composição de uma “teoria do HD”, enquanto outros francamente refutam essa ideia.

Numa meta-análise quantitativa proposta por Rapp e colegas (2012), os autores concluíram que o processamento da linguagem não literal (além de metáforas, foram incluídos também provérbios, expressões idiomáticas, metonímias, ironias e sarcasmo) requer principalmente o recrutamento do hemisfério esquerdo, incluindo o giro frontal inferior, o giro temporal superior e médio e as regiões parietal inferior, pré-central, parahipocampal, cerebelar, frontal superior e pré-frontal média. No HD, houve fortes evidências de ativação do giro frontal inferior.

Como apontam os autores, houve uma forte ativação do $\mathrm{HE}$ no processamento de linguagem figurada. $\mathrm{O}$ giro frontal inferior esquerdo é, para Rapp et al. (2012), a chave para o processamento de linguagem não literal. Segundo os autores, essa região é responsável pelos processos de seleção e de avaliação dos conceitos em jogo nos experimentos.

Com relação ao processamento de metáforas, há algumas considerações específicas a se fazer. Rapp e colegas (2012) selecionaram 17 estudos com linguagem figurada, dentre os quais doze artigos compõem o nosso corpus. Do total analisado pelos autores, um terço não apontou ativação no HD. Nesse um terço, incluemse os seguintes estudos aqui apresentados: Rapp et al. (2004; 2007), Lee e Dapretto (2006), Diaz e Hogstrom (2011). Ou seja, dos vinte estudos que selecionamos, quatro não reportaram significativa ativação do HD no processamento de metáforas. Acrescentamos a esses estudos os achados de Shibata et al. (2007; 2012), Stringaris et al. (2007), Yang et al. (2009) e Mashal et al. (2008), para quem o processamento de metáforas aciona, basicamente, a circuitaria do HE. Para esses autores, não é a metaforicidade a propriedade que requer ativação extra no HD, mas outras questões, como o grau de dificuldade das testagens, por exemplo.

Com relação ao que classificaram como metáforas criativas não salientes, os autores reportaram ativação no $\mathrm{HE}$ dos giros frontal médio, frontal inferior, parahipocampal, frontal medial, temporal médio e occipital médio. No HD, houve apenas ativação no giro frontal inferior. Contribuíram para esses dados sete estudos selecionados pelos autores, todos eles reportados também em nossa seleção.

Houve também referência à ativação de parte do sistema límbico, em especial, do giro parahipocampal (MASHAL et al., 2005; SCHMIDT; SEGER, 2009; YANG et al., 2009; DIAZ et al., 2011), cuja ativação é associada a atividades que requerem processamento de ambiguidades no nível da sentença.

Para os autores, ao final da análise, os dados revelaram que não há força argumentativa nas hipóteses coarse coding e graded salience, porque os dados revelaram pouca ativação no HD, inclusive para elementos linguísticos não convencionais. Há participação do HD no processamento de metáforas, mas não necessariamente um papel crucial, já que não houve consistente menção à circuitaria do HD mencionada nos experimentos em que houve distinção entre metáforas convencionais e metáforas criativas.

Yang e colegas (2014) propuseram um estudo específico sobre a metáfora, com dados coletados até 2011. Foram selecionados dezessete estudos, dos quais treze também fazem parte de nossa seleção.

$\mathrm{Na}$ primeira rodada de análise, na comparação entre processamento da metáfora e processamento de sentenças ou conjunto de palavras literais, os achados indicaram que houve significativa ativação nas seguintes regiões: no HE, giro frontal superior, giro frontal médio, giro frontal inferior, ínsula, giro temporal inferior, giro temporal médio, giro lingual e tálamo; no HD, giro frontal médio, giro frontal inferior, giro temporal médio e giro cingulado.

Mas o que chama atenção na meta-análise proposta por Yang e colegas (2014) é o fato de os pesquisadores terem também dado atenção a questões específicas do processamento da metáfora, como, por exemplo, o efeito da convencionalidade e o efeito da criatividade, tanto em testes de julgamento, quanto em testes de valência.

Os achados dessa meta-análise revelaram que a compreensão de metáforas convencionais implica forte ativação em regiões têmporo-parietais frontais no HE. Entretanto, o processamento de metáforas criativas aumenta a ativação de regiões bilateralmente distribuídas, que incluem o giro frontal inferior direito, a ínsula direita, o giro frontal médio e o giro temporal superior. Para os autores, esses achados confirmam a graded salience hypothesis.

Ainda segundo os autores, o significado de uma metáfora criativa não pode ser acessado diretamente do léxico mental, por não ser um significado saliente, já que os conceitos envolvidos na composição de metáforas criativas são distantes e sem relação semântica direta. 
Dessa forma, o envolvimento do HD nesses casos também confirmaria a coarse-coding hypothesis.

Corroboramos essa conclusão, acrescentando também os achados de Subramanian et al. (2012; 2013) que não foram citados na meta-análise de Yang e colegas, mas foram incluídos em nossa seleção. Esses estudos evidenciaram aumento de ativação bilateralmente distribuída, à medida que se distanciavam semanticamente tópico e veículo.

Outro ponto interessante da meta-análise de Yang e colegas é o fato de os autores terem analisado em separado os estudos sob o ponto de vista da metodologia dos experimentos. Foram agrupados os experimentos que se fundamentaram em testagens no nível da palavra e experimentos com testagens no nível da sentença. Estudos prévios indicavam maior recrutamento do HD no processamento de palavras, enquanto o HE seria responsável pelo processamento de sentenças (FAUST et al., 2003). Entretanto, essa variável não se aplica na meta-análise realizada. Para Yang e colegas, o contraste metáforas em palavras versus metáforas em sentenças implicou maior ativação apenas no giro frontal médio esquerdo, sem significativa ativação no HD. Essa hipótese se confirma nos achados de Lee e Dapretto (2006), para quem o processamento de palavras não resultou maior ativação do HD, e nos achados de Ahrens et al. (2007) e de Schmidt e Seger (2009), para quem o processamento de sentenças não implicou maior ativação do HE. Incluímos, ainda, nesse grupo os estudos de Prat, Mason e Just (2012), cujo experimento se valeu de sentenças apresentadas em pequenos contextos, tendo como resultado ativação da circuitaria do HD.

Aparentemente, o envolvimento do HD no processamento da metáfora se dá de modo mais decisivo em função de outras variáveis além da figuratividade da metáfora. Entender como essas variáveis atuam juntas no processamento da linguagem metafórica parece ser o desafio dos próximos experimentos com fMRI.

Então, dada a diversidade de abordagens e a consequente variedade de achados, é possível pressupor que há algum padrão no processamento de enunciados com metáforas do ponto de vista neurocientífico? A resposta a essa questão parece difícil de delimitar, pois os achados não implicam diretamente um mapeamento anatômico da metáfora no cérebro.

O que parece mais evidente é que talvez a figuratividade per se não seja a propriedade mais importante da metáfora. Aparentemente, a dicotomia literal $\times$ figurado não se concretiza tão especificamente em correlatos neurais no HE e no HD respectivamente. No âmbito da Linguística, essa distinção também gera debates.

Sob que aspectos fundamentos da Linguística poderiam lançar luz ao conjunto de achados? Um caminho possível, em nosso entender, é estabelecer relações interdisciplinares conceituais, uma vez que definições como propriedades semânticas, convencionalidade, não convencionaliade, processamento semântico, processamento pragmático, entre outras, têm natureza linguística. Tendo clareza na abordagem linguística adotada sobre o que é metáfora, o objeto de investigação poderá ser mais bem delimitado, os experimentos mais bem estruturados e, como consequência, talvez seja possível o desenho de uma abordagem da metáfora nas fronteiras entre Neurociência e Linguística.

\section{Considerações finais}

Como pudemos verificar ao longo de nossa análise, parece-nos que a ausência de um diálogo entre Neurociência e Linguística sobre a definição de metáfora e de suas propriedades resulta em uma delimitação problemática de um padrão de processamento da metáfora sob um viés neurológico.

Acreditamos que um caminho mais profícuo seria partir da explicitação de aspectos linguísticos necessários a priori para a configuração dos experimentos com neuroimagem. Entendemos que, nas fronteiras interdisciplinares, perspectivas alternativas podem ser desenhadas para solucionar problemas decorrentes de uma abordagem puramente disciplinar.

\section{Referências}

AHRENS, Kathleen; LIU, Ho-Ling; LEE, Chia-Ying; GONG, Shu-Ping; FANG, Shin-Yi; HSU, Yuan-Yu. Functional MRI of conventional and anomalous metaphors in Mandarin Chinese. Brain and Language, v. 100, n. 2, p. 163-171, fev. 2007.

BAMBINI, Valentina; GENTILI, Claudio; RICCIARDI, Emiliano; BERTINETTO, Pier M.; PIETRINI, Pietro. Decomposing metaphor processing at the cognitive and neural level trough functional magnetic resonance imaging. Brain Research Bulletin, v. 86, n. 3, p. 203-216, out. 2011.

BEEMAN, Mark. Coarse semantic coding and discourse comprehension. In: BEEMAN, Mark; CHIARELLO, Christine (ed.). Right hemisphere language comprehension: perspectives from cognitive neuroscience. Mahwah, NJ: Lawrence Erlbaum, 1998. p. 225-284.

CARDILLO, Eileen R.; WATSON, Christine E.; SCHMIDT, Gwenda L.; KRANJEC, Alexander; CHATTERJEE, Anjan. From novel to familiar: Tuning the brain for metaphors. NeuroImage, v. 59, n. 4, p. 3212-3221, fev. 2012.

DIAZ, Michele T.; BARRET, Kyle T.; HOGTSROM, Larson J. The influence of sentence novelty and figurativeness on brain activity. Neuropsychologia, v. 49, n. 3, p. 320-330, fev. 2011.

DIAZ, Michele T.; HOGSTROM, Larson J. The influence of context on hemispheric recruitment during metaphor processing. Journal of Cognitive Neuroscience, v. 23, n. 11, p. 3586-3597, nov. 2011. 
FERSTL, Evelyn C. The functional neuroanatomy of text comprehension: what's the story so far? In: SCHMALHOFER, Franz; PERFETTI, Charles A. Higher Level Language in the Brain: Inference and Comprehension Processes. London: Psychology Press, 2012. p. 53-102.

FODOR, Jerry A.; PYLYSHYN, Zenon W. Connectionism and cognitive architecture: a critical analysis. Cognition, v. 28, p. 3-71, mar. 1988.

GENTNER, Dedre; BOWDLE, Brian F. Metaphor as structuremapping. In: GIBBS, Raymond W (Ed.). The Cambridge handbook of metaphor and thought. Cambridge: Cambridge University Press, 2008. p. 109-128.

GIORA, Rachel. Understanding figurative and literal language: The graded salience hypothesis. Cognitive Linguistics, v. 8, n. 3, p. 183-206, jan.1997.

GIORA, Rachel, ZAINDEL, Eran, SOROKER, Nachum, BATORI, Gila; KASHER, Asa. Differential effects of rightand left-hemispheric damage on understanding sarcasm and metaphor. Metaphor and Symbol, v. 15, p. 63-83, 2000.

GLUCKSBERG, Sam. How metaphors create categories, quickly. In: GIBBS, Raymond W (Ed.). The Cambridge handbook of metaphor and thought. Cambridge: Cambridge University Press, 2008. p. 67-84.

GLUCKSBERG, Sam; KEYSAR, Boaz. Understanding metaphorical comparisons: beyond similarity. Psychological Review, v. 97, n.1, p. 3-18, jan. 1990.

GLUCKSBERG, Sam; MACGLONE, Matthew; MANFREDI, Deanna. Property attribution in metaphor comprehension. Journal of Memory and Language, v. 36, n. 1, p. 50-67, jan. 1997.

JUNG-BEEMAN, Mark. Bilateral brain processes for comprehending natural language. Trends in Cognitive Science, n. 9, v. 11, p. 712-718, nov. 2005.

JUST, Marcel A.; VARMA, Sashank. The organization of thinking: What functional brain imaging reveals about the neuroarchitecture of complex cognition. Cognitive, Affective \& Behavioral Neuroscience, v. 7, n. 3, p. 153-191, set. 2007.

KASPARIAN, Kristina. Hemispheric differences in figurative language processing: contributions of neuroimaging methods and challenges in reconciling current empirical findings. Journal of Neurolinguistics, v. 26, n. 1, p. 1-21, jan. 2013.

LAIRD, Angela R.; FOX, P. Michele; EICKHOFF, Simon B.; TURNER, Jessica A.; RAY, Kimberly L.; MCKAY, D. Reese; GLAHN, David C.; BECKMANN, Christian F.; SMITH, Stephen M.; FOX, Peter T. Behavioral Interpretations of Intrinsic Connectivity Networks. Fox Journal of Cognitive Neuroscience, v. 23, n. 12, p. 4022-4037, dez. 2011.

LAIRD, Angela R.; FOX, P. Michele; PRICE, Cathy J.; GLAHN, David C.; UECKER, Angela M.; LANCASTER, Jack L.; TURKELTAUB, Peter E.; KOCHUNOV, Peter; FOX, Peter T. ALE meta-analysis: Controlling the false discovery rate and performing statistical contrasts. Human Brain Mapping, v. 25, n. 1 , p. 155-164, maio 2005.

LEE, Susan S.; DAPRETTO, Mirella. Metaphorical vs. literal word meanings: fMRI evidence against a selective role of the right hemisphere. NeuroImage, v. 29, n. 2, p. 536-544, jan. 2006
MASHAL, Nira; FAUST, Miriam; HENDLER, Talma. The role of the right hemisphere in processing nonsalient metaphorical meanings: Application of principal components analysis to fMRI data. Neuropsychologia, v. 43, n. 14, p. 2084-2100, abr. 2005.

MASHAL, Nira; FAUST, Miriam; HENDLER, Talma; JUNGBEEMAN, Mark. An FMRI investigation of the neural correlates underlying the processing of novel metaphoric expressions. Brain and Language, v. 100, n. 2, p. 115-126, fev. 2007.

MASHAL, Nira; FAUST, Miriam; HENDLER, Talma; JUNG-BEEMAN, Mark. An fMRI study of processing novel metaphoric sentences. Laterality, v. 14, n. 1, p. 30-54, jan. 2009.

PRAT, Chantel S.; MASON, Robert A.; JUST, Marcel A. Individual differences in the neural basis of causal inferencing. Brain and Language, v. 116, n. 1, p. 1-13, jan. 2011.

RAPP, Alexander M.; LEUBE, Dirk T.; ERB, Michael; GRODD, Wolfgang; KIRCHER, Tilo T. J. Neural correlates of metaphor processing. Cognitive Brain Research, v. 20, n. 3 , p. 395-402, ago. 2004.

RAPP, Alexander M.; LEUBE, Dirk T.; ERB, Michael; GRODD, Wolfgang; KIRCHER, Tilo T. J. Laterality in metaphor processing: Lack of evidence from functional magnetic resonance imaging for the right hemisphere theory. Brain and Language, v. 100, n. 2, p. 142-149, fev. 2007.

RAPP, Alexander M.; MUTSCHLER, Dorothee E.; ERB, Michael Erb. Where in the brain is nonliteral language? A coordinate-based meta-analysis of functional magnetic resonance imaging studies. NeuroImage, v. 63, n. 1, p. 600 610 , out. 2012.

SHIBATA, Midori; ABE, JJun-ichi; TERAO, Atsushi; MIYAMOTO, Tamaki. Neural mechanisms involved in the comprehension of metaphoric and literal sentences: An fMRI study. Brain Research, v. 1166, p. 92-102, ago. 2007.

SHIBATA, Midori; TOYOMURA, Akira; MOTOYAMA, Hiroki; ITOH, Hiroaki; KAWABATA, Yasuhiro; ABE, Junichi. Does simile comprehension differ from metaphor comprehension? A functional MRI study. Brain and Language, v. 121, n. 3, p. 254-260, jun. 2012.

SCHMIDT, Gwenda; SEGER, Carol A. Neural correlates of metaphor processing: the roles of figurativeness, familiarity and difficulty. Brain and Cognition, v. 71, n. 3, p. 375-386, dez. 2009.

STRINGARIS, Argyris K.; MEDFORD, Nicholas C.; GIAMPIETRO, Vincent; BRAMMER, Michael J.; DAVID, Anthony S. Deriving meaning: Distinct neural mechanisms for metaphoric, literal, and non-meaningful sentences. Brain and Language, v. 100, n. 2, p. 150-162, fev. 2007.

STRINGARIS, Argyris K.; MEDFORD, Nicholas C.; GIORA, Rachel; GIAMPIETRO, Vincent C.; BRAMMER, Michael J.; DAVID, Anthony S. How metaphors influence semantic relatedness judgments: The role of the right frontal cortex. NeuroImage, v. 33, n. 2, p. 784-793, nov. 2006.

SUBRAMANIAM, Karuna; FAUST, Miriam; BEEMAN, Mark; MASHAL, Nira. The repetition paradigm: Enhancement of novel metaphors and suppression of conventional metaphors in the left inferior parietal lobe. Neuropsychologia, v. 50, n. 12, p. 2705-2719, out. 2012 . 
SUBRAMANIAM, Karuna; FAUST, Miriam; BEEMAN, Mark; MASHAL, Nira. Positively valenced stimuli facilitate creative novel metaphoric processes by enhancing medial prefrontal cortical activation. Frontiers in Psychology, v. 4, n. 211, abr. 2013.

YANG, Fanpei G.; EDENS, Jennifer; SIMPSON, Claire; KRAWCZYK, Daniel C. Differences in task demands influence the hemispheric lateralization and neural correlates of metaphor. Brain and Language, v. 111, n. 2, p. 114-124, nov. 2009.
YANG, Jie. The role of the right hemisphere in metaphor comprehension: A meta-analysis of functional magnetic resonance imaging studies. Human Brain Mapping, v. 35, n. 1, p. 107-122, jan. 2014.

Recebido: 30/09/2017

Aprovado: 30/11/2017

Contato:

Kári Lúcia Forneck <kari@univates.br> 